\title{
Definite hermitian forms and the cancellation of simple knots
}

\author{
By \\ EVA BAYER *)
}

Schubert has shown that every classical knot $\Sigma^{1} \subset S^{3}$ factorises uniquely into the connected sum of finitely many indecomposable knots (cf. [12]). In particular cancellation holds for these knots. For higher dimensional simple knots factorisation is not always unique (cf. [5] and [1]), but in many cases we still have cancellation (see [2], Proposition 6.6).

In this note we shall give counter examples to the cancellation of non-singular hermitian and skew-hermitian forms. In order to obtain these examples we shall show that the extension of the $\mathbb{Z}$-lattice $\Gamma_{4 n}, n \neq 1$, to certain orders is indecomposable.

Using the classification of simple $(2 q-1)$-knots $\Sigma^{2 q-1} \subset S^{2 q+1}, q \neq 1$, in terms of $(-1)^{q+1}$-hermitian (Blanchfield) forms, we shall then prove that cancellation does not hold for higher odd-dimensional knots.

I thank Hans-Jochen Bartels and Larry Gerstein for useful conversations.

1. Definite hermitian forms. Let $K$ be a number field with a $Q$-involution which we shall denote by an overbar. Assume that $K$ is totally imaginary and that the fixed field $F$ of the involution is totally real. Let $A$ be an order of $K$, and let $L$ be a torsion free $A$-module of finite rank. We shall say that a hermitian form $h: L \times L \rightarrow A$ is definite if $h$ is anisotropic at every real embedding of $F$. Otherwise we shall say that $h$ is indefinite.

The following is a result of Eichler (cf. [3]).

Lemma 1. Every definite hermitian form decomposes uniquely as an orthogonal sum of indecomposable forms.

Sketch of proof (see Kneser [8] and O'Meara [11], §105). We shall say that $x \in L$ is irreducible if $x$ cannot be written as a sum $x=y+z, y \neq 0, z \neq 0$ and $h(y, z)=0$. Then every $x \in L$ can be expressed as a finite sum of irreducible elements. Indeed, if $x=y+z$ with $h(y, z)=0$ then $h(x, x)=h(y, y)+h(z, z)$. As $h$ is anisotropic at every real place, $h(y, y)$ and $h(z, z)$ have the same sign at each real

*) Supported by the "Fonds National Suisse de la recherche scientifique". 
embedding of $F$. Therefore

$$
N_{K / \mathbb{Q}}(h(y, y))<N_{K / \mathbb{Q}}(h(x, x)) \text {, and } N_{K / \mathbb{Q}}(h(z, z))<N_{K / \mathbb{Q}}(h(x, x)) .
$$

As $N_{K / \mathbb{Q}}(h(x, x))$ is a natural number, we see by induction that $x$ can be written as a finite sum of irreducibles. We shall say that two irreducible elements $x$ and $x^{\prime}$ are equivalent if there exists a finite chain of irreducible elements

$$
x=x_{0}, x_{1}, \ldots, x_{k}=x^{\prime},
$$

such that $h\left(x_{i}, x_{i+1}\right) \neq 0$. Every equivalence class generates a sublattice of $L$, and $L$ is the orthogonal sum of these lattices. It is easy to see (cf. [11], §105), that every orthogonal splitting of $L$ into indecomposables is a permutation of these sublattices.

Let $L$ be a free $\mathbb{Z}$-module of finite rank, and let $b: L \times L \rightarrow \mathbb{Z}$ be a symme:riz $\mathbb{Z}$-bilinear form. Let $\hat{L}=A \otimes_{\mathbb{Z}} L$, and let $h: \hat{L} \times \hat{L} \rightarrow A$ be the hermitian form which is defined by $h(\alpha x, \beta y)=\alpha \bar{\beta} b(x, y)$ for $\alpha, \beta \in A$ and $x, y \in L$. If $b$ is definite then $h$ is also definite.

We shall apply this construction to the $\mathbb{Z}$-bilinear form $b: L \times L \rightarrow \mathbb{Z}$ which corresponds to the lattice $\Gamma_{4 n}$ (cf. [10], chap. II, $\S 6$, or [11], $1106 \mathrm{E}$ ).

Proposition. The hermitian form $A \Gamma_{4 n}$ is indecomposable if $n>1$.

The following lemma is well known.

Lemma 2. Let $m=[F: \mathbb{Q}]$. If $a \in F$ is a totally positive algebraic integer, then $\operatorname{Tr}_{F / \mathbb{Q}}(a) \geqq m$. Moreover, if $\operatorname{Tr}_{F / \mathbb{Q}}(a)=m$ then $a=1$.

This follows immediately from the inequality between arithmetic and geometric means.

Proof of Proposition. Let $V=K e_{1} \oplus \cdots \oplus K e_{4 n}$ with the hermitian form $h\left(e_{i}, e_{j}\right)=\delta_{i j}$. Then $A \Gamma_{4 n}$ is the lattice in $V$ which is generated by $e_{i}+e_{j}$ and $\frac{1}{2}\left(e_{1}+\cdots+e_{4 n}\right)$. We shall prove that if $x \in A \Gamma_{4 n}$ such that $h(x, x)=2$, then $x$ is irreducible.

Indeed, assume that $x=y+z$ with $y \neq 0, z \neq 0$ and $h(y, z)=0$. Therefore $h(x, x)=h(y, y)+h(z, z)$, so we have

$$
2 m=\operatorname{Tr}_{F / \mathbb{Q}}(h(x, x))=\operatorname{Tr}_{F / \mathbb{Q}}(h(y, y))+\operatorname{Tr}_{F / \mathbb{Q}}(h(z, z)),
$$

where $m=[F: \mathbb{Q}]$. But $h(y, y)$ and $h(z, z)$ are both totally positive. By Lemma 2 this implies that $\operatorname{Tr}_{F / Q}(h(y, y))=\operatorname{Tr}_{F / Q}(h(z, z))=m$ (in fact, $h(y, y)=h(z, z)=1$ ). Now we shall show that if $y \in A \Gamma_{4 n}$, then $\operatorname{Tr}_{F / Q}(h(y, y))=m$ is impossible. Indeed, if $y=\sum_{i=1}^{4 n} a_{i} e_{i} \in A \Gamma_{4 n}$, then $a_{i} \in \frac{1}{2} A, a_{i}-a_{j} \in A$ for every $i, j=1, \ldots, 4 n$ and $\sum_{i=1}^{4 n} a_{i} \in 2 A$ (cf. [11], $1106 \mathrm{E}$ ). We have $h(y, y)=\sum_{i=1}^{4 n} a_{i} \bar{a}_{i}$, so $m=\sum_{i=1}^{4 n} \operatorname{Tr}_{F / Q}\left(a_{i} \bar{a}_{i}\right)$. Two cases are possible: either all of the $a_{i}$ 's are in $A$, or $a_{i}=\frac{1}{2} b_{i}$ with $b_{i} \in A$ and $b_{i} \neq 0, i=1, \ldots, 4 n$. If we are in the first case, then Lemma 2 implies that all the $a_{i}$ 's except one, say $a_{1}$, are zero. But then $a_{1} \in 2 A$, which contradicts 
$\operatorname{Tr}_{F / \mathbb{Q}}\left(a_{1} \bar{a}_{1}\right)=m$. In the second case we have $m=1_{4}^{1} \sum_{i=1}^{4 n} b_{i} \bar{b}_{i} \geqq n \cdot m$, using Lemma 2 . But $n>1$ so this is impossible. Let $x_{i}=e_{i}-e_{i+1}$ for $i=1, \ldots, 4 n-1$ and let $x_{4 n}=e_{4 n-1}+e_{4 n}$. We have $h\left(x_{i}, x_{i}\right)=2$, so $x_{1}, \ldots, x_{4 n}$ are irreducible. But $h\left(x_{i}, x_{i+1}\right) \neq 0$, so the $x_{i}$ 's are all in the same indecomposable component of $A \Gamma_{4 n}$ (see Lemma 1). But the $x_{i}$ 's are linearly independent, so this component must be $A \Gamma_{4 n}$.

Remark 1. The proposition can be generalized as follows: If $(L, b)$ is definite, indecomposable, then $(\hat{L}, h)$ is also indecomposable. If $K$ is a quadratic field, then this has been proved by L. Gerstein (cf. [4], Corollary 1.4) and R. Smith (cf. [13], Theorem 2.2). In the general case the analogue of this statement for quadratic forms has been proved by Y. Kitaoka (cf. [7], Corollary of Theorem 4). It is possible to adapt Kitaoka's proof to hermitian forms, only obvious changes are necessary.

Remark 2. Assume that $A$ is integrally closed and that there exists an $\alpha \in A$ such that $\bar{\alpha}+\alpha=1$. Then two indefinite non-singular hermitian forms are isometric if and only if they have the same rank, signatures and isometric determinants (cf. [2], Definition 1.9 and Corollary 4.10).

By contrast, the above proposition shows that the number of isometry classes of definite hermitian forms of rank $4 n$ and determinant $\langle 1\rangle$ is at least $p(n)$, where $p(n)$ is the number of partitions of $n$ into a sum of positive integers. (See Gerstein [4], Theorem 3.9 for related results.)

2. Counter-examples to the cancellation of simple $(2 q-1)$-knots, $q>1$. Let $\lambda \in \mathbb{Z}[x]$ be an irreducible polynomial such that $\lambda(x)=x^{\operatorname{deg} \lambda} . \lambda\left(x^{-1}\right)$ and $\lambda(0)=$ $\lambda(1)=\lambda(-1)=1$.

Set $A=\mathbb{Z}[x] /(\lambda), K=\mathbb{Q}[x] /(\lambda)=\mathbb{Q}(\tau)$. Then $K$ has a $\mathbb{Q}$-involution which sends $\tau$ to $\tau^{-1}$.

Let $M$ be a torsion free $A$-module of finite rank. By results of Kearton, Levine and Trotter, we have: Every non-singular (-1)q+1-hermitian form $h: M \times M \rightarrow A$ can be realized as the Blanchfield form of a simple $(2 q-1)$-knot $\Sigma^{2 q-1} \subset S^{2 q+1}$ if $q>2$. Two simple $(2 q-1)$-knots are isotopic if and only if the associated Blanchfield forms are isometric, for $q>1$ (cf. [6], [9], [14]). Therefore it is enough to show that cancellation does not always hold for non-singular hermitian and skew-hermitian forms.

Let us choose $\lambda$ such that $K$ is totally imaginary and that the fixed field $F$ of the involution is totally real. (For instance, $\lambda(x)=x^{4}-x^{2}+1$, the cyclotomic polynomial corresponding to the 12 th roots of unity.)

We have:

(*) $A \Gamma_{8} \perp A \Gamma_{8} \perp\langle-1\rangle \cong A \Gamma_{16} \perp\langle-1\rangle$

(where $\perp$ denotes orthogonal sum, and $\langle-1\rangle$ is the hermitian form $A e \times A e \rightarrow A$ such that $e e=-1$ ). Indeed, this isomorphism already holds over $\mathbb{Z}$ (cf. [10], 
Chap. II, Theorem (4.3)). On the other hand, $A \Gamma_{8} \perp A \Gamma_{8}$ is not isometric to $A \Gamma_{16}$ because the latter is indecomposable (see Section 1).

This gives the desired counter-example for $q$ odd, $q \neq 1$.

Let $u=\tau-\tau^{-1}$. Then $u$ is a unit of $A$ because

$$
N_{K / \mathbb{Q}}(u)=N_{K / \mathbb{Q}}\left(\tau^{-1}\right) \cdot N_{K / \mathbb{Q}}(\tau-1) N_{K / \mathbb{Q}}(\tau+1)=\lambda(0) \cdot \lambda(1) \cdot \lambda(-1)=1 .
$$

We have $\vec{u}=-u$, so multiplying (*) by $u$ we obtain a counter-example to cancellation of non-singular skew-hermitian forms, i.e. for the case $q$ even, $q \neq 2$.

We need a special argument for 3-knots. Let $h: M \times M \rightarrow A$ be a non-singular skew-hermitian form. There exists a simple 3-knot $\Sigma^{3} \subset S^{5}$ such that the Blanchfield form of $\Sigma^{3}$ is isometric to $h$ if and only if the signature of the intersection form corresponding to $h$ is divisible by 16 (cf. [9], [14]).

Let $\Gamma$ be the orthogonal sum of 16 copies of $\Gamma_{8}$. We have

$$
A \Gamma \perp\langle 1\rangle \perp\langle-1\rangle \cong A \Gamma_{128} \perp\langle 1\rangle \perp\langle-1\rangle \text {. }
$$

As before, we multiply by $u$ in order to obtain skew-hermitian forms.

\section{References}

[1] E. BAYER, Factorisation is not unique for higher dimensional knots. Comment. Math. Helv. $55,583-592(1980)$.

[2] E. BAYER, Unimodular hermitian and skew-hermitian forms. J. Algebra (to appear).

[3] M. EICHLER, Zur Theorie der Kristallgitter. Math. Ann. 125, $51-5 \tilde{5}$ (1952).

[4] L. Gerstenv, Classes of definite hermitian forms. Amer. J. Math. 100, $81-97$ (1978).

[5] C. Kearton, Factorisation is not unique for 3-knots. Indiana Univ. Math. J. 28, 451-452 (1979).

[6] C. Kearton, Blanchfield duality and simple knots. Trans. Amer. Math. Soc. 202, 141-160 (1975).

[7] Y. KrTaoka, Scalar extension of quadratic lattices. Nagoya Math. J. 65. 139-149 (1977).

[8] M. KNeSER, Zur Theorie der Kristallgitter. Math. Ann. 127, 105-106 (1954).

[9] J. LEVINE, An algebraic classification of some knots of codimension two. Comment. Math. Helv. 45, 185-198 (1970).

[10] J. Mrlnor and D. Husemoller, Symmetric bilinear forms. Berlin-Heidelberg-New York 1973.

[11] O. T. O'MEAra, Introduction to quadratic forms. Berlin-Heidelberg-New York 1973.

[12] H. SchUBERT, Die eindeutige Zerlegbarkeit eines Knotens in Primknoten. S.-B. Heidelberger Akad. Wiss. Math.-Natur. Kl. 1, 3, 57-104 (1949).

[13] R. F. SMrrm, The construction of definite indecomposable hermitian forms. Amer. J. Math. 100, 1021-1048 (1978).

[14] H. F. TrotTer, On $S$-equivalence of Seifert matrices. Invent. math. 20, 173-207 (1973).

Eingegangen am 25. 9. 1981

Anschrift des Autors:

Eva Bayer

Université de Genève

Section de Mathématiques

$2-4$, rue du Lièvre

Case postale 124

CH-1211 Genève 24 